\title{
Governança nas Universidades Federais do Brasil segundo o "Levantamento Integrado de Governança Organizacional Pública" do Tribunal de Contas da União
}

Governance in Federal Universities in Brazil According to the Integrated Survey of Public Organizational Governance of the Federal Court of Accounts

\section{Pauliane Venessa da Silva Braga Marques ${ }^{1}$ Maria da Glória Arrais Peter $^{2}$ Cícero Philip Soares do Nascimento ${ }^{3}$ Marcus Vinícius Veras Machado ${ }^{4}$}

\section{RESUMO}

Governar recursos públicos tem sido um grande desafio para o gestor público brasileiro. Nesse contexto, o tema "governança pública" tornou-se um assunto muito relevante nos últimos anos. O objetivo deste estudo foi analisar o nível de adesão das universidades federais brasileiras às boas práticas de governança pública abordadas pelo Tribunal de Contas da União (TCU) em sua mais recente publicação do "Levantamento Integrado de Governança Organizacional Pública", realizada em 2017. A pesquisa tem abordagem descritiva e qualitativa, utilizando-se da análise bibliográfica e documental. $\mathrm{O}$ estudo foi realizado com as sessenta universidades federais que participaram do levantamento. Foram analisados e interpretados os dados fornecidos pelo TCU, mais especificamente, 28 questões do levantamento, relacionadas ao Índice de Governança Pública

1 Mestranda em Políticas Públicas e Gestão da Educação Superior, Universidade Federal do Ceará (UFC). E-mail: pauliane.venessa@gmail.com

2 Doutora em Educação pela Universidade Federal do Ceará (UFC). E-mail: gloria@arrais.com

3 Mestre em Administração e Controladoria pela Universidade Federal do Ceará (UFC). E-mail: philip_nascimento@yahoo.com.br

$4 \quad \mathrm{PhD}$ em Higher Education e Finanças Públicas pela University of Arizona, Estados Unidos. Pró-Reitor de Gestão de Pessoas da Universidade Federal do Ceará (UFC). E-mail: marcusmachado@ufc.br 
(iGovPub). Diante disso, o nível de governança pública das universidades federais brasileiras é considerado "inicial".

Palavras-Chave: Governança Pública. Universidades Federais. Tribunal de Contas da União.

\section{ABSTRACT}

Management of public resources has been a great challenge for Brazilian public managers, which has rendered Public Governance as a particularly relevant subject in recent years. This paper analyzes the level of compliance of Brazilian federal universities with the best practices of Public Governance addressed by the Federal Court of Accounts (TCU) in its most recent publication of the Integrated Survey of Public Organizational Governance, held in 2017. This descriptive/qualitative study used bibliographic and documentary analysis and included 60 federal universities in its survey. The data provided by TCU were analyzed and interpreted, namely 28 survey questions related to the Public Governance Index (iGovPub). The results show that the Public Governance level of Brazilian federal universities in 2017 is considered "initial".

Keywords: Public Governance. Federal Universities. Brazilian Federal Court of Accounts.

Recebido: 22-04-2020

Aprovado: 16-06-2020

\section{INTRODUÇÃO}

A governança vem ganhando abrangência não apenas no setor privado, mas também no setor público, uma vez que os problemas verificados na administração pública podem ser controlados ou amenizados com 
a utilização de alguns princípios oriundos da governança corporativa do setor privado (SLOMSKI et al., 2008).

A governança pública tem assumido grande importância na gestão dos recursos públicos no Brasil, sendo alvo de muitas discussões sobre sua prática. $\mathrm{O}$ valor das boas práticas de governança no setor público vai além de normas e princípios, pois é visto como resultado positivo tanto para a instituição quanto para a sociedade. A governança está presente em todas as esferas públicas: municipal, estadual ou federal.

Nesse ambiente, estão inseridas as instituições federais de ensino superior (Ifes), que, segundo Souza (2017), encontram-se em constante crescimento, tendo em vista as ações de políticas públicas estabelecidas através do Plano de Desenvolvimento da Educação (PDE), criado em 2007, no qual se destaca o Programa de Apoio a Planos de Reestruturação e Expansão das Universidades Federais (Reuni).

A presente pesquisa se destina a uma parcela dessas Ifes, as universidades federais brasileiras. Desse modo, para realização dessas ações de promoção e expansão do ensino superior, é necessária uma boa gestão, que estimule a adoção de melhores práticas, a fim de prover uma maior eficiência para o pleno funcionamento da instituição.

A governança no setor público já foi tema de estudos nacionais e internacionais, como Kuziemski e Misuraca (2020); Rodrigues e Rodrigues (2020); Correio e Correio (2019); Santos e Rover (2019); Teixeira e Gomes (2019); Cappellesso, Figueiredo e Lima (2016); Sukmadilaga, Pratamab e Mulyanic (2015); Matei e Drumasu (2015); Oliveira e Pisa (2015); Silvia e Adela (2014); Madhani (2014); e Benedicto et al. (2013). Já no cenário da governança nas universidades federais brasileiras, tem-se os estudos de Sales et al. (2020); Castello e Pont (2018); Rosini, Messias e Palmisano (2018); Mello e Silva (2018); Castro e Silva (2017); Kelm et al. (2017); e Zorza e Rodrigues (2016).

Ademais, as universidades federais, como órgãos da administração pública federal, fazem parte da lista de organizações que participaram do 
Levantamento de Governança do Tribunal de Contas da União (TCU), em 2017, sobre boas práticas de governança e gestão no setor público.

Assim, em virtude da realização de diversos trabalhos anteriores sobre o tema da governança pública e governança nas universidades federais, surge o seguinte problema: qual o nível de adesão das universidades federais brasileiras às boas práticas de governança pública abordadas pelo TCU no "Levantamento Integrado de Governança Organizacional Pública"? Diante da problemática levantada, e considerando os estudos anteriores sobre a temática, pressupõe-se que as universidades federais brasileiras apresentam baixo nível de maturidade em governança pública.

Para que tal indagação seja respondida, a presente pesquisa tem como objetivo geral analisar o nível de adesão das universidades federais brasileiras às boas práticas de governança pública abordadas pelo TCU no referido levantamento. Para atingir o objetivo proposto, foram delimitados os seguintes objetivos específicos: (1) identificar as universidades federais brasileiras com maior adesão às práticas de governança; (2) verificar as práticas de governança pública mais adotadas de acordo com o levantamento; e (3) identificar qual mecanismo de Governança - liderança, estratégia ou accountability - tem maior aderência pelas universidades federais brasileiras.

Considerando o objetivo proposto, esta pesquisa pode ser definida como aplicada e descritiva, tendo como procedimentos metodológicos a pesquisa bibliográfica e documental. Para a coleta de dados, foram utilizadas as informações contidas no site do TCU, compreendendo a mais recente publicação do "Levantamento Integrado de Governança Organizacional Pública”, realizado em 2017. Além disso, solicitou-se por e-mail de contato do órgão a identificação das universidades federais que participaram do levantamento realizado em 2017 sobre governança pública.

Tendo em vista a importância da governança e do levantamento realizado pelo TCU, que busca identificar as práticas de governança utilizadas na administração pública federal, a pesquisa torna-se relevante para 
o fortalecimento da confiança da gestão nas instituições públicas. Além disso, visa identificar as necessidades da sociedade e ampliar os resultados esperados, assegurando ao cidadão que os recursos públicos estão sendo administrados da melhor forma possível.

O trabalho está estruturado em cinco seções, sendo a primeira a presente introdução, em que são apresentadas as diretrizes condutoras da pesquisa. $\mathrm{Na}$ segunda seção, tem-se a discussão teórica acerca da governança no setor público; a terceira apresenta os procedimentos metodológicos adotados para a condução da pesquisa; na quarta expõe-se a consolidação dos resultados; e, por fim, na sexta seção, apresentam-se as conclusões do estudo.

\section{REFERENCIAL TEÓRICO}

\subsection{Governança pública e universidades federais brasileiras}

A governança corporativa emergiu como um conjunto de práticas adotadas pelas organizações no contexto de separação entre propriedade e gestão das grandes empresas contemporâneas (MARTINS et al. 2016; OLIVEIRA; FONTES FILHO, 2017). Seu objetivo era criar mecanismos de redução da assimetria informacional e do problema de agência (agency), caracterizado, segundo a teoria da agência (JENSEN; MECKLING, 1976), como o ambiente de conflitos de interesses no qual os acionistas buscam agregar valor à empresa e os executivos buscam a longevidade da organização e o aumento do próprio poder (ANDRADE, 2008; MARTINS et al. 2016; SILVA, 2014).

Partindo desse cenário, a redução de conflitos de interesse por meio da governança corporativa faz uso de mecanismos como transparência (disclosure), prestação de contas (accountability) e equidade (fairness) (ANDRADE, 2008; MARTINS et al. 2016), que ganham relevância no contexto das organizações públicas, na medida em que se apresentam como instrumentos de fortalecimento das relações entre governo e sociedade (MATIAS-PEREIRA, 2010). 
Matei e Drumasu (2015) afirmam que o modelo de governança corporativa foi adotado e implementado no setor público como uma nova abordagem de gestão pública nas décadas de 1980 e 1990, por países como Nova Zelândia, Austrália e Reino Unido.

Sobre esse aspecto, Benedicto et al. (2013) afirmam que, assim como ocorreu o movimento internacional no âmbito das empresas, a adoção de práticas de governança no setor público vem se aprimorando e buscando adaptações para o seu contexto e necessidades.

Para Edwards et al. (2012), a emergência do modelo de governança pública ancora-se na interseção e crescentes conexões entre os setores público e privado nas últimas três décadas, comportamento que tem sido uma dimensão fundamental para compreensão da governança no setor público.

Buscando esclarecer o papel da governança nas organizações, diversas entidades publicaram estudos buscando estabelecer padrões de boas práticas, como Organização para a Cooperação Desenvolvimento Econômico (ORGANIZATION FOR ECONOMIC CO-OPERATION AND DEVELOPMENT, 2015), a Australian National Audit Office (2014), a Internacional Federation of Accountants (Ifac) (INTERNATIONAL FEDERATION OF ACCOUNTANTS; PUBLIC FINANCE AND ACCOUNTANCY, 2014), e o TCU (BRASIL, 2014).

O estudo denominado "International framework: good governance in the public sector", da Ifac (2014), afirma que a governança no setor público compreende os arranjos estabelecidos de modo a garantir o alcance dos objetivos e metas dos agentes, permitindo que as entidades atinjam os resultados de interesse público pretendidos (MATEI; DRUMASU, 2015).

Cappellesso, Figueiredo e Lima (2016) afirmam que o framework apresentado pela Ifac (2014) evidencia um esforço dos envolvidos no estudo em reduzir o conflito de agência por meio de boas práticas de governança no contexto das organizações públicas.

Oliveira e Fontes Filho (2017) acrescentam que, no âmbito do Estado, mais especificamente na administração pública indireta, há diversas 
relações de agência, protagonizadas por meio de contratos tácitos ou explícitos entre cidadãos, políticos, Estado, sistema econômico e burocracia.

Nesse ambiente, Sales et al. (2020) argumentam que as universidades federais brasileiras, que pertencem à administração pública sob a forma de autarquias ou fundações, estão sujeitas ao conjunto normativo que rege o setor público. Embora a Constituição Federal de 1988 tenha conferido autonomia às universidades, estas estão sujeitas à rigidez da legislação, que envolve a definição da estrutura organizacional e o controle da aplicação dos recursos (SALES et al., 2020). Observa-se, então, que as universidades federais brasileiras, assim como o setor público, necessitam do estabelecimento de mecanismos de governança que permitam transparência, equidade e prestação de contas, uma vez que utilizam recursos públicos.

Sobre esse aspecto, os governos têm implementado novos modelos de gestão nas Instituições de Ensino Superior (IES), procurando alterar o paradigma vigente nas universidades que estão dentro da esfera pública (ARAÚJO; RODRIGUES, 2009; SALES et al., 2020). Tal afirmação está em sintonia com Benedicto et al. (2013) e Edwards et al. (2012), para quem os modelos de gestão e governança no setor público procuram fazer uma conexão com a matriz estabelecida pelas organizações privadas.

Assim, Araújo e Rodrigues (2009) afirmam que o caminho da gestão universitária para um novo modelo ancora-se naquele proposto pela teoria da agência (JENSEN; MECKLING, 1976), ao permitir que as universidades (as agências) tomem decisões sobre a implementação de programas com base na economia e eficiência, em detrimento das organizações políticas.

Mello e Silva (2018) afirmam que, tendo em vista o cenário socioeconômico vigente e estudos anteriores, os desafios enfrentados pelas IES dizem respeito à capacidade de estruturar e manter uma gestão, fazendo uso de práticas de governança capazes de otimizar o desempenho e proteger os stakeholders, além de, simultaneamente, proporcionar benefícios aos processos internos. 
Desta forma, a governança pública no contexto das universidades federais brasileiras apresenta-se como um cenário de grandes desafios, o que enseja a realização de estudos que investiguem como as boas práticas de governança estão sendo aplicadas nas IES brasileiras. Com efeito, as universidades federais brasileiras, como órgãos da administração pública federal, fazem parte da lista de organizações que participaram do levantamento sobre boas práticas de governança e gestão no setor público realizado pelo TCU.

\subsection{Governança no contexto do TCU}

O TCU atua na esfera administrativa julgando as contas de administradores públicos e demais responsáveis por dinheiro, bens e valores públicos federais, bem como as contas de qualquer pessoa que causem prejuízo ao erário.

De acordo com a Constituição Federal de 1988, o TCU auxiliará o Congresso Nacional no exercício do Controle Externo (BRASIL, 1988). A missão institucional do TCU (BRASIL, 2015, p. 44) é "aprimorar a administração pública em benefício da sociedade, através do controle externo". O TCU exerce algumas funções básicas, como fiscalizar, consultar, informar, judiciar, sancionar, corrigir e normatizar, além da atividade de ouvidoria, havendo, ainda, em sua atuação, o propósito de educar.

Nos últimos anos, o TCU tem buscado contribuir para o aprimoramento da governança no setor público através de publicações relacionadas ao tema, como a publicação do "Referencial Básico de Governança" (BRASIL, 2014), aplicável a órgãos e entidades da administração pública.

O TCU (BRASIL, 2014) define governança no setor público como um conjunto de mecanismos de liderança, estratégia e controle que objetivam avaliar, direcionar e monitorar a gestão, para a condução de políticas e prestação de serviços de interesse da sociedade. Esses mecanismos não produzem os resultados separadamente. Para a obtenção dos resultados 
esperados, foi criado, no "Referencial Básico de Governança”, quatro níveis de análise, a saber: (1) os mecanismos de governança, (2) os componentes, (3) as práticas e (4) os itens de controle. A Figura 1 apresenta os componentes dos mecanismos de governança.

Figura 1: Componentes dos mecanismos de governança

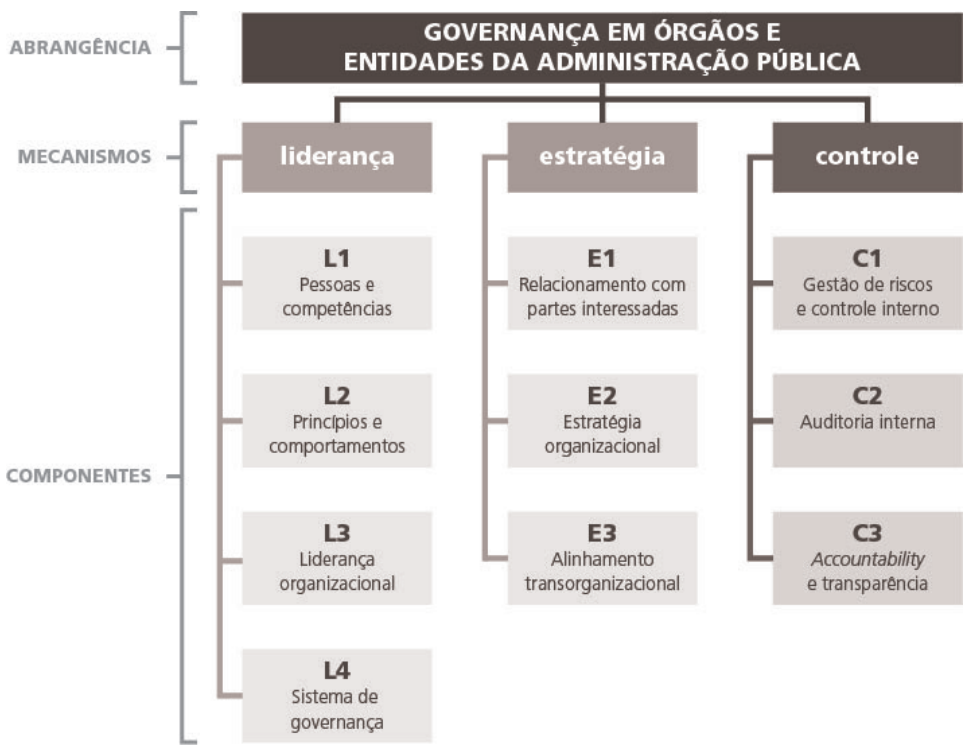

Fonte: Tribunal de Contas da União (BRASIL, 2014, p. 39).

Conforme Figura 1, os líderes das organizações são os responsáveis por conduzir o processo de estabelecimento dos mecanismos de governança, no qual a liderança relaciona-se com o conjunto de práticas de natureza humana; a estratégia abrange a definição e monitoramento de objetivos, indicadores e metas, bem como o alinhamento entre planos e operações de unidades e organizações envolvidas na sua execução; e o controle, por sua vez, abrange aspectos como transparência, prestação de contas e responsabilização, dentre outros (BRASIL, 2014). 
Cada um dos mecanismos de governança está associado a um conjunto de componentes que, por sua vez, foram vinculados a um grupo de práticas de governança, em que contribuem, direta ou indiretamente, para o alcance dos objetivos da instituição. A estrutura evidenciada na Figura 1 serve como base para algumas das questões relacionadas ao tema governança pública do "Levantamento Integrado de Governança Organizacional Pública", do TCU.

O TCU vem realizando, nos últimos anos, levantamentos de governança e gestão em quatro temas distintos - (1) tecnologia da informação, (2) pessoas, (3) contratações e (4) governança pública -, com a finalidade de conhecer melhor a situação da governança no setor público e estimular as organizações públicas a adotarem boas práticas de governança.

Em 2017, o órgão reuniu esses temas e realizou o primeiro "Levantamento Integrado de Governança Organizacional Pública”, com a participação de organizações públicas que compõem a administração direta e indireta da esfera federal ou que recebem recursos públicos federais (BRASIL, 2018). Dentre as organizações, participaram 113 instituições de ensino, sendo sessenta universidades federais, objeto de investigação do presente estudo.

O questionário que compõe o levantamento no ciclo de 2017 é composto por 93 perguntas e está fundamentado em diversas referências nacionais e internacionais de boas práticas de governança, em normas vigentes e, ainda, em recomendações do TCU. Divide-se em cinco temas: (1) governança pública (liderança, estratégia, accountability); (2) governança e gestão de pessoas; (3) governança e gestão de tecnologia da informação; (4) governança e gestão de contratações; e (5) resultados. Com a análise dos resultados obtidos, foi apresentado, por meio de "perfis", o panorama da governança pública na administração pública federal, dentre eles: perfil integrado de governança e gestão; perfil de governança pública; perfil de governança e gestão de pessoas; perfil de governança e gestão de TI; perfil de governança e gestão de contratações e perfil resultados. 
O foco do presente estudo foi o perfil de governança pública, representado pelo Índice de Governança Pública (iGovPub), que resulta das questões relacionadas ao tema governança pública, que abrange os mecanismos de governança: liderança, estratégia e accountability.

2.3 Evidências empíricas sobre governança nas universidades federais públicas

A governança no setor público foi objeto de estudo em diferentes situações em âmbito nacional e internacional. Diversos pesquisadores publicaram estudos internacionais sobre investigação da governança no contexto das entidades públicas, como Kuziemski e Misuraca (2020), que discutiram a governança e inteligência artificial no setor público; Sukmadilaga, Pratamab e Mulyanic (2015), que investigaram a implementação da boa governança por meio da análise da divulgações de demonstrações financeiras; Matei e Drumasu (2015), que realizaram uma análise acadêmica do processo de desenvolvimento do conceito de governança corporativa em entidades públicas e como essa é uma forma eficiente de governança; Silvia e Adela (2014), que estudaram a transparência no setor público baseada na governança eletrônica; e Madhani (2014), que discutiram a evolução das reformas de governança corporativa do setor público.

Em âmbito nacional, Rodrigues e Rodrigues (2020) analisaram a relação entre a governança pública e as reeleições municipais no contexto mineiro; Correio e Correio (2019) investigaram as práticas de governança pública adotadas por 372 órgãos da administração pública federal brasileira; Santos e Rover (2019) verificaram a influência de práticas de governança pública na eficiência da aplicação dos recursos públicos em educação e saúde nos municípios brasileiros; Teixeira e Gomes (2019) desenvolveram uma revisão teórica da literatura sobre o conceito de governança pública; Cappellesso, Figueiredo e Lima (2016) analisaram a contribuição dos novos padrões contábeis para as práticas de governança 
corporativa no setor público brasileiro; Oliveira e Pisa (2015) estudaram o desenvolvimento de um índice de medição da governança pública a partir do ponto de vista dos princípios de governança; e Benedicto et al. (2013) analisaram a aplicabilidade dos conceitos da governança corporativa na administração pública diante do contexto atual, que demanda eficiência, responsabilidade, ética, transparência e prestação de contas.

Já sobre as boas práticas de governança nas universidades federais brasileiras, Sales et al. (2020) investigaram o nível de aderência dessas instituições às práticas de governança pública recomendadas pelo "Study 13", da Ifac, considerando as dimensões: estrutura e processos organizacionais e controle. Concluiu-se que o nível de aderência das universidades é cerca de $49 \%$ do total de 77 recomendações analisadas.

Castello e Pont (2018) objetivaram realizar o constructo teórico referente às práticas de governança pública em IES a partir da abordagem sistêmica, tendo como objeto a Universidade Federal do Pará (UFPA). Os resultados apontaram para o ineditismo da análise de governança relacionada à abordagem sistêmica e às constatações de carência de pesquisa de governança nas universidades da Amazônia.

Rosini, Messias e Palmisano (2018) analisaram a percepção dos avaliadores que compõem o quadro de avaliadores do Instituto Nacional de Estudos e Pesquisas Educacionais Anísio Teixeira (Inep), tendo como base a concepção de boas práticas de governança nas IES. Os resultados evidenciam aspectos positivos no processo de avaliação, fortalecendo a governança das instituições em todas as suas perspectivas.

Mello e Silva (2018) investigaram as práticas de governança corporativa utilizadas em uma fundação de ensino superior comunitária à luz das melhores práticas definidas pelo Instituto Brasileiro de Governança Corporativa (IBGC) (2015). Observou-se que a entidade adota um grande número das práticas de governança corporativa propostas pelo IBGC, adaptando algumas delas de forma a atender suas especificidades. 
Castro e Silva (2017) avaliaram a prestação de contas do exercício de 2014 de cinco universidades públicas federais brasileiras de acordo com as boas práticas de governança, usando um quadro avaliativo composto por práticas publicadas por órgãos e pesquisadores. Os resultados mostraram que as universidades alcançaram em média $45,7 \%$ de aderência ao quadro proposto, o que indica a necessidade de maiores investimentos na adoção de boas práticas de governança.

Kelm et al. (2017) realizaram um debate das características típicas das instituições comunitárias de ensino superior (Ices), a partir dos conflitos de agência e dos desdobramentos dos interesses conflitantes e, às vezes, difusos de diversos atores que a compõem.

Por fim, o estudo realizado por Zorza e Rodrigues (2016) pesquisou o nível de transparência e boas práticas de governança dos relatórios de gestão de cinco universidades federais brasileiras que estavam no topo do Ranking Webmetrics, em julho de 2013. Os resultados demonstraram que nenhuma das universidades analisadas atingiu o patamar de alta transparência, classificando-as no nível médio de transparência.

\section{METODOLOGIA}

A pesquisa científica é procedimento racional e sistemático, com vistas a proporcionar respostas aos problemas inicialmente formulados, sendo desenvolvida por meio dos conhecimentos disponíveis e da utilização cuidadosa de métodos, técnicas e outros procedimentos científicos, ou seja, para se realizar uma pesquisa é necessário seguir uma metodologia (GIL, 2002).

Assim, quanto a sua natureza, a pesquisa é classificada como aplicada, pois, conforme Silva (2005), objetiva gerar conhecimentos para aplicação prática destinada à solução de problemas específicos. No caso, pretende-se saber o nível de adesão das universidades federais brasileiras às boas práticas de governança pública abordadas pelo TCU no mais recente "Le- 
vantamento Integrado de Governança Organizacional Pública", sob o enfoque dos mecanismos de governança: liderança, estratégia e accountability.

Em relação aos objetivos gerais, pode-se classificá-la como descritiva, pois, de acordo com Gil (2002), essas pesquisas descrevem características de determinada população ou fenômeno ou, então, estabelece relações entre variáveis.

A população estudada são sessenta universidades federais brasileiras que participaram da mais recente edição do referido levantamento, em 2017 (Quadro 1). 
Quadro 1: Universidades federais brasileiras participantes do levantamento de governança do TCU

\begin{tabular}{|c|c|c|}
\hline Região & Universidade & UF \\
\hline \multirow{5}{*}{ Centro-Oeste (5) } & Universidade de Brasília (UnB) & $\mathrm{DF}$ \\
\hline & Universidade Federal de Goiás (UFG) & GO \\
\hline & Universidade Federal da Grande Dourados (UFGD) & MS \\
\hline & Universidade Federal de Mato Grosso (UFMT) & MT \\
\hline & Universidade Federal de Mato Grosso do Sul (UFMS) & MS \\
\hline \multirow{18}{*}{ Nordeste (18) } & Universidade Federal de Alagoas (Ufal) & $\mathrm{AL}$ \\
\hline & Universidade Federal da Bahia (Ufba) & $\mathrm{BA}$ \\
\hline & Universidade Federal do Oeste da Bahia (Ufob) & $\mathrm{BA}$ \\
\hline & Universidade Federal do Recôncavo da Bahia (UFRB) & $\mathrm{BA}$ \\
\hline & Universidade Federal do Sul da Bahia (UFSB) & $\mathrm{BA}$ \\
\hline & $\begin{array}{l}\text { Universidade da Integração Internacional da Lusofonia Afro-Bra- } \\
\text { sileira (Unilab) }\end{array}$ & $\mathrm{CE}$ \\
\hline & Universidade Federal do Cariri (UFCA) & $\mathrm{CE}$ \\
\hline & Universidade Federal do Ceará (UFC) & $\mathrm{CE}$ \\
\hline & Universidade Federal da Paraíba (UFPB) & PB \\
\hline & Universidade Federal de Campina Grande (UFCG) & $\mathrm{PB}$ \\
\hline & Universidade Federal de Pernambuco (UFPE) & $\mathrm{PE}$ \\
\hline & Universidade Federal Rural de Pernambuco (UFRPE) & PE \\
\hline & Universidade Federal do Rio Grande do Norte (UFRN) & $\mathrm{RN}$ \\
\hline & Universidade Federal Rural do Semi-Árido (Ufersa) & $\mathrm{RN}$ \\
\hline & Universidade Federal de Sergipe (UFS) & SE \\
\hline & Universidade Federal do Maranhão (Ufma) & MA \\
\hline & Universidade Federal do Piauí (UFPI) & PI \\
\hline & Universidade Federal do Vale do São Francisco (Univasf) & PE \\
\hline
\end{tabular}


Quadro 1: Universidades federais brasileiras participantes do levantamento de governança do TCU (continuação)

\begin{tabular}{|c|c|c|}
\hline \multirow{4}{*}{ Norte (10) } & Universidade Federal de Rondônia (Unir) & RO \\
\cline { 2 - 3 } & Universidade Federal do Amapá (Unifap) & AP \\
\cline { 2 - 3 } & Universidade Federal do Tocantins (UFT) & TO \\
\cline { 2 - 3 } & Universidade Federal do Oeste do Pará (Ufopa) & PA \\
\cline { 2 - 3 } & Universidade Federal do Pará (UFPA) & PA \\
\hline & Universidade Federal do Sul e Sudeste do Pará (Unifesspa) & PA \\
\hline Universidade Federal Rural da Amazônia (Ufra) & PA \\
\hline Universidade Tecnológica Federal do Paraná (UTFPR) & PA \\
\hline Universidade Federal de Roraima (UFRR) & RR \\
\hline Universidade Federal do Amazonas (Ufam) & AM \\
\hline Universidade Federal da Integração Latino-Americana (Unila) & PR \\
\hline Universidade Federal do Paraná (UFPR) & PR \\
\hline Universidade Federal da Fronteira Sul (Uffs) & RS \\
\hline Universidade Federal de Santa Maria (UFSM) & RS \\
\hline & Universidade Federal do Rio Grande do Sul (UFRGS) & RS \\
\hline Universidade Federal de Santa Catarina (UFSC) & SC \\
\hline & Universidade Federal do Pampa (Unipampa) & RS \\
\hline & Universidade Federal do Rio Grande (Furg) & RS \\
\hline
\end{tabular}


Quadro 1: Universidades federais brasileiras participantes do levantamento de governança do TCU (continuação)

\begin{tabular}{|c|c|c|}
\hline \multirow{4}{*}{} & Universidade Federal de Alfenas (Unifal) & MG \\
\cline { 2 - 3 } & Universidade Federal de Itajubá (Unifei) & MG \\
\cline { 2 - 3 } & Universidade Federal de Juiz de Fora (UFJF) & MG \\
\cline { 2 - 3 } & Universidade Federal de Lavras (Ufla) & MG \\
\cline { 2 - 3 } & Universidade Federal de Minas Gerais (UFMG) & MG \\
\cline { 2 - 3 } & Universidade Federal de Uberlândia (UFU) & MG \\
\hline Universidade Federal do Triângulo Mineiro (UFTM) & MG \\
\hline Universidade Federal dos Vales do Jequitinhonha e Mucuri & MG \\
\cline { 2 - 3 } & Universidade Federal do Estado do Rio de Janeiro (Unirio) & RJ \\
\hline Universidade Federal do Rio de Janeiro (UFRJ) & RJ \\
\hline Universidade Federal Rural do Rio de Janeiro (UFRRJ) & RJ \\
\hline Universidade Federal de São Paulo (Unifesp) & SP \\
\hline Universidade Federal de Ouro Preto (Ufop) & MG \\
\hline Universidade Federal de São Carlos (UFSCar) & SP \\
\hline Universidade Federal De São João Del Rei (UFSJ) & MG \\
\hline Universidade Federal de Viçosa (UFV) & MG \\
\hline & SP \\
\hline
\end{tabular}

Fonte: Elaborado pelos autores (2018).

Quanto aos procedimentos, como foram utilizadas as respostas fornecidas pelas universidades ao questionário do TCU, a pesquisa é considerada documental, e os documentos classificados como "de segunda mão", que, conforme Gil (2008), são aqueles que de alguma forma já foram analisados, tais como: relatórios de pesquisa, relatórios de empresas, tabelas estatísticas, entre outros. A pesquisa fez uso de 28 questões relacionadas ao conjunto "Governança Pública: liderança, estratégia e accountability", conforme o Quadro 2. 
Quadro 2: Questões sobre o tema governança pública do Levantamento de Governança Pública do TCU

\begin{tabular}{|c|c|}
\hline \multicolumn{2}{|r|}{ Mecanismo 1100. Liderança } \\
\hline Práticas & Questões \\
\hline \multirow{2}{*}{$\begin{array}{l}\text { 1110. Estabelecer o modelo de } \\
\text { governança da organização }\end{array}$} & $\begin{array}{l}\text { 1111. A estrutura interna de governança da organização está } \\
\text { definida. (tipo M) }\end{array}$ \\
\hline & $\begin{array}{l}\text { 1112. Há segregação de funções para tomada de decisões } \\
\text { críticas. (tipo A) }\end{array}$ \\
\hline \multirow{2}{*}{$\begin{array}{l}\text { 1120. Gerir o desempenho da } \\
\text { alta administração }\end{array}$} & $\begin{array}{l}\text { 1121. A seleção de membros da alta administração é feita com } \\
\text { base em critérios e procedimentos estabelecidos. (tipo A) }\end{array}$ \\
\hline & $\begin{array}{l}\text { 1122. O desempenho de membros da alta administração é } \\
\text { avaliado. (tipo A) }\end{array}$ \\
\hline \multirow{2}{*}{$\begin{array}{l}\text { 1130. Zelar por princípios de } \\
\text { ética e conduta. }\end{array}$} & $\begin{array}{l}\text { 1131. Código de ética e de conduta aplicável aos membros } \\
\text { de conselho ou colegiado superior e aos membros da alta } \\
\text { administração da organização está estabelecido. (tipo A) }\end{array}$ \\
\hline & $\begin{array}{l}\text { 1132. Os casos de conflitos de interesse, envolvendo pessoa } \\
\text { que pertença à alta administração ou ao conselho ou colegiado } \\
\text { superior, são identificados e tratados. (tipo A) }\end{array}$ \\
\hline \multicolumn{2}{|r|}{ Mecanismo 2100. Estratégia } \\
\hline Práticas & Questões \\
\hline \multirow{2}{*}{$\begin{array}{l}\text { 2110. Gerir os riscos da } \\
\text { organização. }\end{array}$} & $\begin{array}{l}\text { 2111. O modelo de gestão de riscos da organização está } \\
\text { estabelecido. (tipo M) }\end{array}$ \\
\hline & $\begin{array}{l}\text { 2112. Os riscos considerados críticos para a organização são } \\
\text { geridos. (tipo A) }\end{array}$ \\
\hline \multirow{4}{*}{$\begin{array}{l}\text { 2120. Estabelecer a estratégia } \\
\text { da organização }\end{array}$} & $\begin{array}{l}\text { 2121. O modelo de gestão estratégica da organização está } \\
\text { estabelecido. (tipo M) }\end{array}$ \\
\hline & 2122. A estratégia da organização está definida. (tipo E) \\
\hline & $\begin{array}{l}\text { 2123. Os principais processos estão identificados e mapeados. } \\
\text { (tipo A) }\end{array}$ \\
\hline & $\begin{array}{l}\text { 2124. As demandas das partes interessadas estão identificadas, } \\
\text { mapeadas e priorizadas. (tipo A) }\end{array}$ \\
\hline
\end{tabular}


Quadro 2: Questões sobre o tema governança pública do Levantamento de Governança Pública do TCU (continuação)

\begin{tabular}{|c|c|}
\hline Práticas & Questões \\
\hline \multirow{8}{*}{$\begin{array}{l}\text { 2130. Promover a gestão } \\
\text { estratégica }\end{array}$} & $\begin{array}{l}\text { 2131. A alta administração estabeleceu modelo de gestão dos } \\
\text { processos finalísticos. (tipo M) }\end{array}$ \\
\hline & $\begin{array}{l}\text { 2132. A alta administração monitora o desempenho da gestão } \\
\text { dos processos finalísticos. (tipo A) }\end{array}$ \\
\hline & $\begin{array}{l}\text { 2133. A alta administração estabeleceu modelo de gestão de } \\
\text { pessoas. (tipo M) }\end{array}$ \\
\hline & $\begin{array}{l}\text { 2134. A alta administração monitora o desempenho da gestão } \\
\text { de pessoas. (tipo A) }\end{array}$ \\
\hline & $\begin{array}{l}\text { 2135. A alta administração estabeleceu modelo de gestão de } \\
\text { tecnologia da informação. (tipo M) }\end{array}$ \\
\hline & $\begin{array}{l}\text { 2136. A alta administração monitora o desempenho da gestão } \\
\text { de tecnologia da informação. (tipo A) }\end{array}$ \\
\hline & $\begin{array}{l}\text { 2137. A alta administração estabeleceu modelo de gestão de } \\
\text { contratações. (tipo M) }\end{array}$ \\
\hline & $\begin{array}{l}\text { 2138. A alta administração monitora o desempenho da gestão } \\
\text { de contratações. } \\
\text { (tipo A) }\end{array}$ \\
\hline \multicolumn{2}{|r|}{ Mecanismo 3100. Accountability } \\
\hline Práticas & Questões \\
\hline \multirow{4}{*}{$\begin{array}{l}\text { 3110. Promover transparência, } \\
\text { responsabilidade e prestação de } \\
\text { contas. }\end{array}$} & 3111. O modelo de transparência está estabelecido. (tipo M) \\
\hline & $\begin{array}{l}\text { 3112. O modelo de prestação de contas diretamente à } \\
\text { sociedade está estabelecido. (tipo M) }\end{array}$ \\
\hline & $\begin{array}{l}\text { 3113. O modelo de responsabilização está estabelecido. (tipo } \\
\text { M) }\end{array}$ \\
\hline & $\begin{array}{l}\text { 3114. A organização publica conjuntos de dados de forma } \\
\text { aderente aos princípios de dados abertos. (tipo A) }\end{array}$ \\
\hline \multirow{4}{*}{$\begin{array}{l}\text { 3120. Assegurar a efetiva atua- } \\
\text { ção da auditoria interna }\end{array}$} & $\begin{array}{l}\text { 3121. A organização definiu o estatuto da auditoria interna. } \\
\text { (tipo E) }\end{array}$ \\
\hline & $\begin{array}{l}\text { 3122. A organização elabora Plano Anual de Auditoria Interna. } \\
\text { (tipo A) }\end{array}$ \\
\hline & $\begin{array}{l}\text { 3123. A auditoria interna produz relatórios destinados às ins- } \\
\text { tâncias internas de governança. (tipo A) }\end{array}$ \\
\hline & $\begin{array}{l}\text { 3124. A organização avalia o desempenho da função de audito- } \\
\text { ria interna com base em indicadores e metas. (tipo A) }\end{array}$ \\
\hline
\end{tabular}

Fonte: Adaptado de TCU (BRASIL, 2018). 
De acordo com o relatório técnico detalhado disponibilizado pelo TCU em seu site, a categorização das respostas do questionário e, consequentemente, dos índices divulgados, foram definidos em estágios de capacidade, da seguinte forma: a) 0,4 é o limite inferior da faixa intermediária - assim as respostas com valores menores que $40 \%$ foram classificadas no estágio inicial -; b) valor 0 (zero) para o limite inferior da faixa inicial e, dentro dessa faixa, os valores menores que $15 \%$ foram classificados como estágio inexpressivo; c) valor de $70 \%$ para o limite superior da faixa intermediária - então, as respostas acima desse valor foram classificadas na faixa aprimorada -; e d) valor 1, como limite superior da faixa aprimorada. A Tabela 1 demonstra a categorização das respostas.

Tabela 1: Categorização das respostas por estágios de capacidade

\begin{tabular}{c|c|c}
\hline \multicolumn{2}{c|}{ Estágios } & Intervalos \\
\hline \multirow{2}{*}{ Inicial } & Inexpressivo & 0 a $14,99 \%$ \\
\cline { 3 - 4 } & Iniciando & 15 a $39,99 \%$ \\
\hline \multicolumn{2}{c|}{ Intermediário } & 40 a $70 \%$ \\
\hline \multicolumn{2}{c|}{ Aprimorado } & 70,01 a $100 \%$ \\
\hline
\end{tabular}

Fonte: Adaptado de TCU (BRASIL, 2018).

Com a categorização por estágios de capacidade, é possível identificar como se apresenta a maturidade em governança das universidades federais investigadas.

\section{ANÁLISE DOS RESULTADOS}

Para responder à pergunta norteadora da pesquisa, foram analisadas as respostas do questionário aplicado no "Levantamento Integrado de Governança Organizacional Pública”, ciclo 2017, fornecidas pelo TCU, sob o tema "governança pública", composto por três mecanismos: liderança, estratégia e accountability. 
4.1 Aderência das Universidades Federais às práticas de Governança Pública

Para verificar a aderência das universidades federais brasileiras às práticas de governança pública com o enfoque do levantamento de governança pública aplicado pelo TCU, foi elaborado um ranking do iGovPub, que agrega os resultados referentes às questões sobre os mecanismos: liderança, estratégia e accountability, conforme demonstrado na Tabela 2.

Tabela 2: Aderência das universidades federais às práticas de governança pública

\begin{tabular}{|c|c|c|c|c|c|}
\hline Ranking & Universidade & iGovPub & $32^{\circ}$ & Universidade & iGovPub \\
\hline $1^{\mathrm{o}}$ & UFMS & 0,80 & $33^{\circ}$ & UFG & 0,38 \\
\hline $2^{\circ}$ & UFRPE & 0,60 & $34^{\circ}$ & UFRB & 0,37 \\
\hline $3^{\circ}$ & Unifal & 0,59 & $21^{\circ}$ & UFTM & 0,36 \\
\hline $4^{\circ}$ & Ufla & 0,58 & \multirow{2}{*}{$22^{\circ}$} & UFRR & 0,35 \\
\hline $5^{\circ}$ & Univasf & 0,57 & & Ufam & 0,35 \\
\hline $6^{\circ}$ & Ufra & 0,54 & \multirow{3}{*}{$23^{\circ}$} & UFABC & 0,34 \\
\hline $7^{\circ}$ & UFV & 0,53 & & UNIR & 0,34 \\
\hline \multirow{2}{*}{$8^{\circ}$} & Unifap & 0,51 & & Ufersa & 0,34 \\
\hline & UnB & 0,51 & $24^{\circ}$ & UFSM & 0,33 \\
\hline \multirow{4}{*}{$9^{\circ}$} & UFPel & 0,50 & \multirow{2}{*}{$25^{\circ}$} & UFS & 0,32 \\
\hline & Unifesp & 0,50 & & UFCSPA & 0,32 \\
\hline & Unipampa & 0,50 & $26^{\circ}$ & UFSB & 0,31 \\
\hline & UFMT & 0,50 & \multirow{2}{*}{$27^{\circ}$} & UFCG & 0,30 \\
\hline $10^{\circ}$ & UFFS & 0,48 & & UFJF & 0,30 \\
\hline $11^{\circ}$ & UFCA & 0,47 & $28^{\circ}$ & UFRJ & 0,29 \\
\hline $12^{\circ}$ & Furg & 0,46 & \multirow{4}{*}{$29^{\circ}$} & UFGD & 0,28 \\
\hline \multirow{2}{*}{$13^{\circ}$} & UFPB & 0,45 & & Unilab & 0,28 \\
\hline & UFPI & 0,45 & & Ufob & 0,28 \\
\hline \multirow{4}{*}{$14^{\circ}$} & UFT & 0,44 & & UFPE & 0,28 \\
\hline & UFSJ & 0,44 & \multirow{2}{*}{$30^{\circ}$} & UFSC & 0,25 \\
\hline & UFPA & 0,44 & & UFC & 0,25 \\
\hline & Ufopa & 0,44 & $31^{\circ}$ & UFRRJ & 0,23 \\
\hline
\end{tabular}


Tabela 2: Aderência das universidades federais às práticas de governança pública (continuação)

\begin{tabular}{|c|c|c|c|c|c|}
\hline $15^{\circ}$ & UFU & 0,43 & $32^{\circ}$ & Ufba & 0,22 \\
\hline \multirow{2}{*}{$16^{\circ}$} & UFRN & 0,42 & $33^{\circ}$ & UFRGS & 0,20 \\
\hline & Ufop & 0,42 & $34^{\circ}$ & Ufal & 0,19 \\
\hline \multirow{2}{*}{$17^{\circ}$} & UFMG & 0,40 & \multirow{2}{*}{$35^{\circ}$} & Unila & 0,18 \\
\hline & UFVJM & 0,40 & & UFPR & 0,18 \\
\hline $18^{\circ}$ & UFSCar & 0,39 & \multirow{3}{*}{$36^{\circ}$} & Unirio & 0,13 \\
\hline \multirow{2}{*}{$19^{\circ}$} & Unifesspa & 0,38 & & UTFPR & 0,13 \\
\hline & Unifei & 0,38 & & UFMA & 0,13 \\
\hline
\end{tabular}

Fonte: Elaborado pelos autores (2018).

De acordo com o iGovPub, foi possível elaborar um ranking com as universidades, dividindo-as em colocações do primeiro ao $36^{\circ}$ lugar. A Universidade Federal de Mato Grosso do Sul (UFMS) destaca-se por seu elevado índice de representatividade em governança pública junto às demais universidades federais.

$\mathrm{Na}$ Tabela 3, apresenta-se a estatística descritiva dos dados em relação à quantidade de universidades federais brasileiras que aderem em maior quantidade às práticas de boa governança.

Tabela 3: Estatística descritiva da aderência das universidades as práticas de governança pública

\begin{tabular}{c|c|c|c}
\hline Mínimo & Máximo & Média & Desvio padrão \\
\hline 0,13 & 0,80 & 0,38 & 0,133065803 \\
\hline
\end{tabular}

Fonte: Elaborado pelos autores (2018).

Verificou-se, na Tabela 3, que houve diferença entre o índice mínimo $(0,13)$ e o máximo $(0,80)$, que se refere a aderência às práticas de governança pública nas universidades federais, demonstrando uma disparidade entre as universidades em relação ao nível aprimorado de governança e ao nível inicial. Através do desvio padrão (0,133065803), observa-se, ainda, a existência de uma dispersão relevante entre os valores expressos na amostra, confirmando a situação em que os dados apresentados possuem dispersão distante da média, o que reforça a diferença de desempenho das universidades. 
Conforme o relatório técnico detalhado divulgado pelo TCU, foi possível demonstrar, através da Tabela 4, as universidades por estágios de capacidade (inexpressivo, inicial, intermediário e aprimorado), com base na Tabela 1, do tema "governança pública".

Tabela 4: Relação das universidades federais por estágios de capacidade do iGovPub

\begin{tabular}{|c|c|c|c|c|c|}
\hline \multicolumn{2}{|c|}{ Estágios } & Intervalos & Universidades & Quantidade & $\%$ \\
\hline \multirow[b]{2}{*}{ Inicial } & Inexpressivo & 0 a $14,99 \%$ & Unirio, UTFPR, UFMA & 3 & $5 \%$ \\
\hline & Iniciando & 15 a $39,99 \%$ & $\begin{array}{l}\text { UFSCar, Unifesspa, Unifei, } \\
\text { UFG, UFRB, UFTM, UFRR, } \\
\text { Ufam, UFABC, Unir, Ufersa, } \\
\text { UFSM, UFS, UFCSPA, } \\
\text { UFSB, UFCG, UFJF, UFRJ, } \\
\text { UFGD, Unilab, Ufob, UFPE, } \\
\text { UFSC, UFC, UFRRJ, Ufba, } \\
\text { UFRGS, Ufal, Unila, UFPR }\end{array}$ & 30 & $50 \%$ \\
\hline \multicolumn{2}{|c|}{ Intermediário } & 40 a $70 \%$ & $\begin{array}{c}\text { UFRPE, Unifal, Ufla, } \\
\text { Univasf, Ufra, UFV, Unifap, } \\
\text { UnB, UFPel, Unifesp, } \\
\text { Unipampa, UFMT, UFFS, } \\
\text { UFCA, Furg, UFPB, UFPI, } \\
\text { UFT, UFSJ, UFPA, Ufopa, } \\
\text { UFU, UFRN, Ufop, UFMG, } \\
\text { UFVJM }\end{array}$ & 26 & $43,33 \%$ \\
\hline \multicolumn{2}{|c|}{ Aprimorado } & $\begin{array}{c}70,01 \mathrm{a} \\
100 \%\end{array}$ & UFMS & 1 & $1,66 \%$ \\
\hline
\end{tabular}

Fonte: Elaborado pelos autores (2018).

Nota-se que a única instituição que alcança o estágio aprimorado é a Universidade Federal de Mato Grosso do Sul (UFMS). Percebe-se que $43,33 \%$ das universidades se encontram no estágio intermediário e 55\% no estágio inicial de governança pública.

Diante do resultado do iGovPub apresentado na Tabela 4, conclui-se que o nível de governança das universidades federais pesquisadas é considerado inicial, corroborando, portanto, os resultados dispostos nos trabalhos citados na seção 3 deste estudo, em que é demonstrado um nível abaixo da média no quesito boas práticas de governança nas universidades. 
4.2 Aderência das práticas de governança pública nas universidades federais brasileiras

Para identificar as práticas de governança pública mais aderentes nas universidades, foi elaborado, a partir do Quadro 2, um ranking com o resultado da média das respostas das questões correspondentes às práticas (Tabela 5).

Tabela 5: Ranking das práticas relacionadas à governança pública mais aderente nas universidades federais

\begin{tabular}{c|l|c}
\hline Ranking & \multicolumn{1}{|c}{ Prática } & Índice \\
\hline $1^{\circ}$ & 1130: Zelar por princípios de ética e conduta & 0,54 \\
\hline $2^{\circ}$ & 1110: Estabelecer o modelo de governança da organização & 0,52 \\
\hline $3^{\circ}$ & 3120: Assegurar a efetiva atuação da Auditoria Interna & 0,48 \\
\hline $4^{\circ}$ & 3110: Promover transparência, responsabilidade e prestação de contas & 0,45 \\
\hline $5^{\circ}$ & 2120: Estabelecer a estratégia da organização & 0,43 \\
\hline $6^{\circ}$ & 2130: Promover a gestão estratégica & 0,29 \\
\hline $7^{\circ}$ & 1120: Gerir o desempenho da alta administração & 0,27 \\
\hline $8^{\circ}$ & 2110: Gerir os riscos da organização & 0,12 \\
\hline
\end{tabular}

Fonte: Elaborado pelos autores (2018).

Conforme Tabela 5, as práticas mais adotadas referem-se ao mecanismo liderança ( $1^{\circ}$ e $2^{\circ}$ lugares), seguido da accountability ( $3^{\circ}$ e $4^{\circ}$ lugares $)$.

4.3 Mecanismo de Governança Pública em maior destaque nas Universidades Federais

Com o resultado de cada bloco de questões correspondentes aos mecanismos de governança, foi realizada a média aritmética para a obtenção do mecanismo de maior aderência, explicitado na Tabela 6 . 
Tabela 6: Média dos Mecanismos de Governança Pública

\begin{tabular}{|c|c|c|c|}
\hline Universidade & 0,45 & Estratégia & Accountability \\
\hline UFMS & 0,38 & 0,58 & 0,94 \\
\hline UFRPE & 0,19 & 0,46 & 0,60 \\
\hline Unifal & 0,22 & 0,47 & 0,76 \\
\hline Ufla & 0,12 & 0,41 & 0,72 \\
\hline Univasf & 0,37 & 0,34 & 0,83 \\
\hline Ufra & 0,27 & 0,37 & 0,47 \\
\hline UFV & 0,32 & 0,45 & 0,52 \\
\hline Unifap & 0,32 & 0,43 & 0,77 \\
\hline UnB & 0,35 & 0,43 & 0,35 \\
\hline UFPel & 0,66 & 0,24 & 0,72 \\
\hline Unifesp & 0,51 & 0,47 & 0,53 \\
\hline Unipampa & 0,37 & 0,10 & 0,75 \\
\hline UFMT & 0,37 & 0,29 & 0,69 \\
\hline UFFS & 0,25 & 0,47 & 0,64 \\
\hline UFCA & 0,38 & 0,23 & 0,68 \\
\hline Furg & 0,47 & 0,37 & 0,44 \\
\hline UFPB & 0,17 & 0,52 & 0,37 \\
\hline UFPI & 0,24 & 0,20 & 0,39 \\
\hline UFT & 0,14 & 0,50 & 0,51 \\
\hline UFSJ & 0,16 & 0,29 & 0,58 \\
\hline UFPA & 0,23 & 0,27 & 0,55 \\
\hline Ufopa & 0,13 & 0,19 & 0,60 \\
\hline UFU & 0,17 & 0,30 & 0,54 \\
\hline UFRN & 0,18 & 0,36 & 0,28 \\
\hline Ufop & 0,28 & 0,30 & 0,46 \\
\hline UFMG & 0,30 & 0,35 & 0,21 \\
\hline UFVJM & 0,11 & 0,15 & 0,32 \\
\hline UFSCar & 0,14 & 0,20 & 0,58 \\
\hline Unifesspa & 0,42 & 0,29 & 0,53 \\
\hline Unifei & 0,40 & 0,17 & 0,58 \\
\hline UFG & 0,37 & 0,28 & 0,49 \\
\hline
\end{tabular}


Tabela 6: Média dos Mecanismos de Governança Pública (continuação)

\begin{tabular}{|c|c|c|c|}
\hline Universidade & 0,45 & Estratégia & Accountability \\
\hline UFRB & 0,45 & 0,23 & 0,44 \\
\hline UFTM & 0,38 & 0,17 & 0,53 \\
\hline UFRR & 0,19 & 0,37 & 0,49 \\
\hline Ufam & 0,22 & 0,23 & 0,58 \\
\hline UFABC & 0,12 & 0,30 & 0,60 \\
\hline Unir & 0,37 & 0,12 & 0,53 \\
\hline Ufersa & 0,27 & 0,20 & 0,54 \\
\hline UFSM & 0,32 & 0,24 & 0,43 \\
\hline UFS & 0,32 & 0,28 & 0,37 \\
\hline UFCSPA & 0,35 & 0,19 & 0,41 \\
\hline UFSB & 0,66 & 0,08 & 0,20 \\
\hline UFCG & 0,51 & 0,14 & 0,25 \\
\hline UFJF & 0,37 & 0,19 & 0,33 \\
\hline UFRJ & 0,37 & 0,02 & 0,49 \\
\hline UFGD & 0,25 & 0,19 & 0,42 \\
\hline Unilab & 0,38 & 0,14 & 0,33 \\
\hline Ufob & 0,47 & 0,19 & 0,19 \\
\hline UFPE & 0,17 & 0,14 & 0,53 \\
\hline UFSC & 0,24 & 0,09 & 0,41 \\
\hline UFC & 0,14 & 0,12 & 0,47 \\
\hline UFRRJ & 0,16 & 0,14 & 0,39 \\
\hline Ufba & 0,23 & 0,08 & 0,35 \\
\hline UFRGS & 0,13 & 0,25 & 0,22 \\
\hline Ufal & 0,17 & 0,11 & 0,29 \\
\hline Unila & 0,18 & 0,19 & 0,18 \\
\hline UFPR & 0,28 & 0,01 & 0,24 \\
\hline Unirio & 0,30 & 0,06 & 0,04 \\
\hline UTFPR & 0,11 & 0,10 & 0,18 \\
\hline UFMA & 0,14 & 0,11 & 0,13 \\
\hline Média & 0,42 & 0,25 & 0,47 \\
\hline
\end{tabular}

Fonte: Elaborado pelos autores (2018). 
Constata-se, a partir da Tabela 6, que o mecanismo de governança pública de maior adesão nas universidades federais é accountability (47\%), seguido de liderança (42\%) e estratégia (25\%). A Tabela 7 apresenta a estatística descritiva dos mecanismos de maior adesão.

Tabela 7: Estatística descritiva dos mecanismos de maior adesão nas universidades federais

\begin{tabular}{c|c|c|c|c}
\hline Mecanismo & Mínimo & Máximo & Média & Desvio padrão \\
\hline Liderança & 0,11 & 0,88 & 0,42 & 0,195530869 \\
\hline Estratégia & 0,01 & 0,58 & 0,25 & 0,136500136 \\
\hline Accountability & 0,04 & 0,94 & 0,47 & 0,185507526 \\
\hline
\end{tabular}

Fonte: Elaborado pelos autores (2018).

Verifica-se, na Tabela 7, uma grande diferença entre os índices mínimos e os índices máximos de ambos os mecanismos pesquisados, reforçando, através do desvio padrão, a existência de uma alta dispersão entre as universidades, constatando, assim, a heterogeneidade dos dados.

\section{CONCLUSÃO}

Para a obtenção do objetivo da pesquisa, foi realizada tabulação dos dados divulgados pelo TCU no levantamento em seu site e a identificação das universidades federais participantes.

Verificou-se que, das sessenta universidades que responderam ao questionário do levantamento, apenas a UFMS atingiu o estágio aprimorado em governança pública, sendo a primeira colocada no ranking de aderência às práticas de governança pública.

Quanto às práticas de governança pública mais adotadas pelas universidades, constatou-se que a prática "zelar por princípios de ética e conduta" é a de maior aderência nas universidades federais, seguida de "estabelecer o modelo de governança da organização", ambas pertencentes ao mecanismo "liderança".

Quanto aos mecanismos de governança pública, observou-se que 
accountability é o que mais se destaca, possivelmente em virtude da Lei $n^{\circ}$ 12.527/2011 (Lei de Acesso à Informação - LAI).

O nível de governança pública da maioria das universidades federais brasileiras, no caso 55\%, é considerado estágio inicial, sob o enfoque do tema governança pública do Levantamento Integrado de Governança Organizacional Pública, do TCU, ciclo 2017, confirmando, assim, o pressuposto da pesquisa.

Pretende-se que o conhecimento produzido com base nos resultados desta pesquisa seja utilizado pelas universidades federais brasileiras a fim contribuir para o aperfeiçoamento de suas funções, além de servir como referência a uma comparação junto às demais IES.

Propõe-se, para pesquisas futuras, um estudo completo dos temas abordados no levantamento, bem como a inclusão das demais IES, com a finalidade de realizar uma análise mais abrangente sobre a governança no setor público.

\section{REFERÊNCIAS}

ANDRADE, G. A. R. de. Estudo econométrico dos efeitos da migração para OIGC: índice de ações com governança corporativa diferenciada da Bovespa. Internext, São Paulo, v. 3, n. 1, p. 39-53, 2008.

ARAÚJO, J. F. F. E.; RODRIGUES, I. M. C. Novos modelos de gestão das universidades: desafios emergentes. In: COLÓQUIO INTERNACIONAL SOBRE GESTÃO UNIVERSITÁRIA NA AMÉRICA DO SUL, 9., 2009, Florianópolis. Anais [...]. Florianópolis: UFSC, 2009.

AUSTRALIAN NATIONAL AUDIT OFFICE. Public sector governance: strengthening performance through good governance. Canberra: Australian National Audit Office, 2014. 
BENEDICTO, S. C. et al. Governança corporativa: uma análise da aplicabilidade dos seus conceitos na administração pública. Organizações Rurais \& Agroindustriais, Lavras, v. 15, n. 2, p. 286-300, 2013.

BRASIL. Tribunal de Contas da União. Referencial básico de governança aplicada a órgãos e entidades da administração pública. Brasília, DF: TCU, 2014.

BRASIL. Constituição da República Federativa do Brasil. Brasília, DF: Senado, 1988.

BRASIL. Tribunal de Contas da União. Plano estratégico do Tribunal de Contas da União 2015-2021. Brasília, DF: TCU, 2015. Disponível em: https://bit.ly/2yxlIpW. Acesso em: 1 ago. 2018.

BRASIL. Tribunal de Contas da União. Resultados do Levantamento Integrado de Governança Organizacional Pública. Brasília, DF: TCU, 2018. Disponível em: https://bit.ly/3eK0w0O. Acesso em: 3 ago. 2018.

CAPPELLESSO, G.; FIGUEIREDO, L. M., LIMA, D. V. A contribuição dos novos padrões contábeis para a governança corporativa do setor público brasileiro. Racef, Ribeirão Preto, v. 7, n. 3, p. 77-90, 2016.

CASTELLO, R.; PONT, J. A governança nas instituições de ensino superior: discussões teóricas e a aplicação na Universidade Federal do Pará. Gigapp Estudios Working Papers, Madrid, v. 87, n. 1, p. 168-184, 2018.

CASTRO; C. J.; SILVA, G. V. Boas práticas de governança aplicadas no setor público: uma análise na prestação de contas de cinco universidades públicas federais. Revista Espacios, Caracas, v. 38, n. 17, 2017. 
CORREIO, M. N. O. P.; CORREIO, O. V. O. Práticas de Governança Pública Adotadas pela Administração Pública Federal Brasileira. Administração Pública e Gestão Social, Viçosa, MG, v. 11, n. 2, p. 215-231, 2019.

EDWARDS, M. et al. Public sector governance in Australia. Canberra: Australian National University E Press, 2012.

GIL, Antônio Carlos. Como elaborar projetos de pesquisas. 4 ed. São Paulo: Atlas, 2002.

GIL, Antônio Carlos. Métodos e téenicas de pesquisa social. 6. ed. São Paulo: Atlas, 2008.

INSTITUTO BRASILEIRO DE GOVERNANÇA CORPORATIVA. Código das melhores práticas de governança corporativa. 5 ed. São Paulo: IBGC, 2015.

INTERNATIONAL FEDERATION OF ACCOUNTANTS; PUBLIC FINANCE AND ACCOUNTANCY. International framework: good governance in the public sector. [S. 1.]: Ifac: CIPFA, 2014.

JENSEN, M. C.; MECKLING, W. H. Theory of the firm: managerial behavior, agency costs and ownership structure. Journal of Financial Economics, v. 3, p. 305-360, 1976.

KELM, M. L. et al. Conflitos de agência e os desafios da governança em uma universidade comunitária. In: COLÓQUIO INTERNACIONAL DE GESTÃO UNIVERSITÁRIA, 18., 2017, Mar del Plata. Anais [...]. Mar del Plata: Universidad Nacional de Mar del Plata, 2017. p. 1-17.

KUZIEMSKI, M.; MISURACA, G. AI governance in the public sector: three 
tales from the frontiers of automated decision-making in democratic settings. Telecommunications Policy, [s. 1.], v. 44, n. 6, p. 1-13, jul. 2020. Elsevier BV. MADHANI, P. Corporate governance and disclosure: public sector vs private sector. SCMS Journal of Indian Management, [s. 1.], v. 11, n. 1, p. 5-20, 2014.

MARTINS, V. A. et al. Teoria de agência aplicada no setor público. Revista Espacios, Caracas, v. 37, n. 35, p. 24, 2016.

MATEI, A.; DRUMASU, C. Corporate governance and public sector entities. Procedia Economics And Finance, [s. 1.], v. 26, p. 495-504, 2015.

MATIAS-PEREIRA, J. A governança corporativa aplicada no setor público brasileiro. Administração Pública e Gestão Social, Viçosa, MG, v. 2, n. 1, p. 109-134, 2010.

MELLO, M. S.; SILVA, R. O. Práticas de governança corporativa em instituições de ensino superior: um estudo de caso em uma universidade de Santa Catarina. Revista de Administração e Contabilidade, Santo Ângelo, v. 33, n. 17, p. 68-80, 2018.

OLIVEIRA, A. G.; PISA, B. J. IGovP: índice de avaliação da governança pública: instrumento de planejamento do Estado e de controle social pelo cidadão. Revista de Administração Pública, Rio de Janeiro, v. 49, n. 5, p. 1263-1290, 2015.

OLIVEIRA, C.; FONTES FILHO, J. R. Problemas de agência no setor público: o papel dos intermediadores da relação entre poder central e unidades executoras. Revista de Administração Pública, Rio de Janeiro, v. 51, n. 5, p. 596-615, 2017.

ORGANIZATION FOR ECONOMIC CO-OPERATION AND DEVELOPMENT. OECD guidelines on corporate governance of state-owned 
enterprises. Paris: OECD, 2015.

RODRIGUES, A. C.; RODRIGUES, S. B. Governança pública e reeleições municipais em Minas Gerais. Administração Pública e Gestão Social, Viçosa, MG, v. 12, n. 1, p. 1-18, 2020.

ROSINI, A. M.; MESSIAS, J. F.; PALMISANO, A. Impacto das Avaliações de Curso do INEP como Instrumento de Governança nas IES no Brasil. Repae: Revista Ensino e Pesquisa em Administração e Engenharia, São Paulo, v. 4, n. 1, p. 115-131, 2018.

SALES, E. C. A. S. et al. Governança no setor público segundo a IFAC - estudo nas Universidades Federais Brasileiras. Brazilian Journal of Business, Curitiba, v. 2, n. 2, p. 1477-1495, 2020.

SANTOS, R. R. D.; ROVER, S. Influência da governança pública na eficiência da alocação dos recursos públicos. Revista de Administração Pública, Rio de Janeiro, v. 53, n. 4, p. 732-752, 2019.

SILVA, A. L. C. da. Governança corporativa e sucesso empresarial: melhores práticas para aumentar o valor da firma. 2. ed. São Paulo: Saraiva, 2014.

SILVA, E. L.; MENEZES, Estera Muszkat. Metodologia da pesquisa e elaboração de dissertação. - 4. ed. rev. atual. Florianópolis: UFSC, 2005.

SILVIA, N. C.; ADELA, D. Romanian public sector transparency approached by e-governance. Procedia Economics And Finance, [s. 1.], v. 15, p. 414-420, 2014. DOI 10.1016/s2212-5671(14)00470-5.

SLOMSKI, V. et al. Governança corporativa e governança da gestão 
pública. São Paulo: Atlas, 2008.

SOUZA, W. M. Eficiência relativa da governança de tecnologia da informação nas instituições federais de ensino superior do Brasil sob a perspectiva da gestão de TI. 2017. 141 f. - Dissertação (Mestrado Políticas Públicas e Gestão da Educação Superior) - Universidade Federal do Ceará, Fortaleza, 2017.

SUKMADILAGA, C.; PRATAMA, A.; MULYANI, S. Good governance implementation in public sector: exploratory analysis of government financial statements disclosures across ASEAN countries. Procedia: Social And Behavioral Sciences, [s. 1.], v. 211, p. 513-518, 2015.

TEIXEIRA, A. F.; GOMES, R. C. Governança pública: uma revisão conceitual. Revista do Serviço Público, Brasília, DF, v. 70, n. 4, p. 519-550, 2019.

ZORZA, L.; RODRIGUES, G. M. Transparência e boas práticas de governança: análise de padrões e princípios nos relatórios de gestão de Universidades Federais brasileiras. In: ENCONTRO NACIONAL DE PESQUISA EM CIÊNCIA DA INFORMAÇÃO, 17., 2016, Salvador. Anais [...]. Salvador: Ancib, 2016. p. 1-22. 